\title{
Design of a tablet holder with the help of Axiomatic Design International Conference of Axiomatic Design 2017
}

\author{
Auðunn Herjólfsson ${ }^{1}$, Haraldur Helgason ${ }^{1}$, Sindri S. Ingvason ${ }^{1}$, Práinn Pórarinsson ${ }^{\text {, }}$, and Joseph Timothy Foley ${ }^{1, \star ~}$ \\ ${ }^{1}$ Reykjavik University, Menntavegur 1, Reykjavik 101, Iceland
}

\begin{abstract}
With the explosion of smart devices, tablets can currently be found everywhere. From schools to kiosks to watching movies in bed, these devices are prevalent everywhere in modern life. The problem with watching movies in bed using tablets is the necessity of hand usage. The market currently holds a few products that attempt to solve this, but none truly frees the user, allowing them hands-free usage with an easy exit of the bed. In this paper, we will describe a design which, utilizing axiomatic design, will out-perform anything currently existing in the same field, by giving a stable viewing experience while fitting to nearly any bed or sofa. Axiomatic Design ensured a comprehensive design process by ensuring customer needs were transformed into carefully thought out functional requirements and design parameters while maintaining modularity.
\end{abstract}

\section{Introduction}

Media has over the years become one of most popular recreational activity in the modern home. Not too long ago it was common to have a 2nd TV set in the bedroom but with increased usage in smart devices such as phones or tablets, they are becoming fewer. Watching a movie in bed or net-surfing is something most people can relate to in one way or another. The problem with the tablet is to gain a good viewing angle. This problem even existed with the TV sets as people tried to attach them near the ceiling. With the tablet, being a smaller object, people often hold it above their head, leading to bloodless arms or lay it on their knees leading to great neck pain. The Recreational Entertainment and Comfort-Enhancer, or TREC-E for short, aims to solve this 1st world problem with a unit resulting in a hands-free viewing experience ideal for anyone to catch up on his favorite series before sleep.

\subsection{Customer Needs}

For a design to happen, the first step is to accurately define the needs of the customer. And the first step of defining the needs of the customer is to realize who the customer is, so the product can be designed for the right person. The goals of identifying customer needs are, according to Ulrich and Eppinger [1], as follows:

- Ensure that the product is focused on customer needs.

- Identify latent or hidden needs as well as explicit needs.

- Provide a fact base for justifying the product specifications.

- Create an archival record of the needs activity of the development process

\footnotetext{
^e-mail: foley@ru.is
}

- Ensure that no critical customer need is missed or forgotten.

- Develop a common understanding of customer needs among members of the development team.

The customer of this product is a person who likes to watch movies or other media while lying down on a bed or a sofa. To assess the customer needs, the team had a brainstorming session to try and figure out the necessities of the product, considering the base idea and said customer.

- $\mathrm{CN}_{1}$ Fit on as many different beds and couches as possible

- $\mathrm{CN}_{2}$ Hold tablets/other smart devices of various size and weight

- $\mathrm{CN}_{3}$ Push easily out of the way when the user is in need of getting up

- $\mathrm{CN}_{4}$ Adjust easily while remaining stable

\section{Prior Art}

There are many types of tablet holders on the market. Even after narrowing them down into those who are suitable for the bedroom there are a only a few major concepts.

\subsection{Similar Patents}

A search of patents involving the fixturing and positioning of tablets to a surface results in a large variety of approaches.

- The "Tablet Holder", a device which seems to be designed for the purpose of holding tablets, but not mounting them to anything [2] 


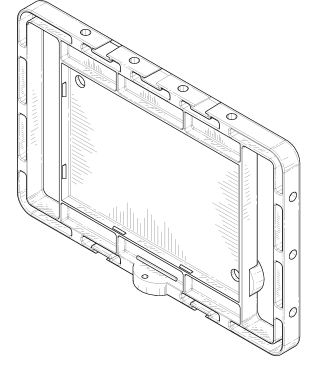

Fig. 1. The patented "Tablet Holder", as pictured in the patent. Image taken from Google patents.

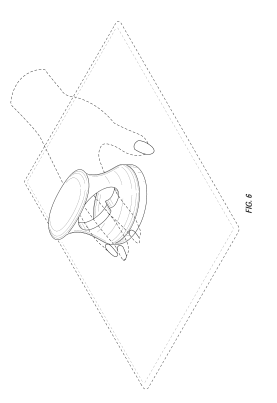

Fig. 2. The patented "Computer Tablet Holder", as pictured in the patent. Image taken from Google patents.

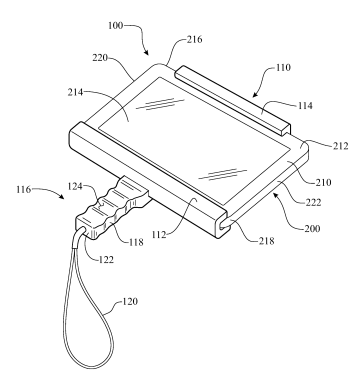

Fig. 3. The patented "Multi Media Tablet Holder", as pictured in the patent. Image taken from Google patents.

- The "Computer Tablet Holder", a device for gripping the tablet in a comfortable way, but requires a manual holding [3]

- The "Multi-Media Tablet Holder", similar to the "Tablet Holder", but with a piece of string, presumably to hang the device for hands-free usage [4]

- The oddly-named "Sqweezel", which is mentioned in this list despite only having a patent pending, is a custom-order product, for hands-free usage. It is available in several colors and a few different shapes, all utilizing a rubber clamp to fit a multitude of devices, and either a table mount or permanent wall mount. [5]

- The "Tabbi" is yet another concept and boasts the ability to attach to nearly any surface, through the utilization of a patented gel suction cup.

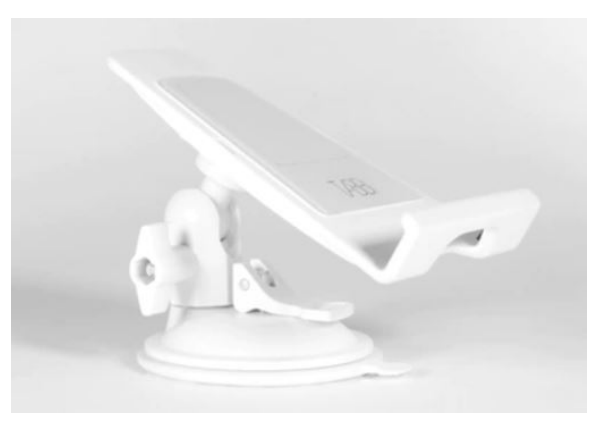

Fig. 4. The Tabbi, with the patented gel suction cup, as pictured on the official Kickstarter page. Image taken from Kickstarter.

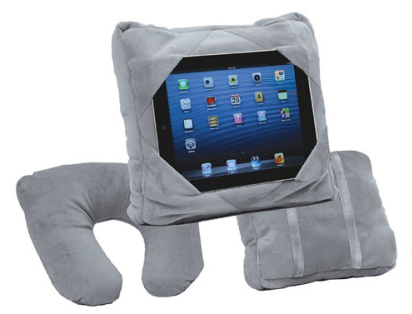

Fig. 5. GOGO Pillow [6]

\subsection{Commercially available tablet fixtures}

All of the market-available concepts do free the hands for other activities but do so in quite different ways. From resting on the mattress along with the user to merging into a pillow or clamping to tables, the goal always seems to be stability along with hands-free usage.

\subsubsection{GOGO Pillow}

One example is the GOGO Pillow shown in Fig. 5, with retail value from amazon.co.uk at $43.14 £$. Some of its notable features and limitations include:

1. Can only be placed at a relatively low viewing angle

2. A pillow resting on a bed is highly stable

The GOGO pillow is highly limited when it comes to the $\mathrm{CN}_{4}$, as possible viewing positions are very few.

\subsubsection{Tablift}

Another concept is based on the idea of crouching over its user and therefore does not leave much room for moving around. For the same reason, it might be a tiring process to move it out of the way in a need of getting up. The four-legged design does not provide a variety of viewing positions and is limited to quite a low viewing angle. An example of this is the Tablift shown in Fig. 6 which sells at $\$ 39.95$ on www.tablift.com.

Some of its notable features and limitations include: 


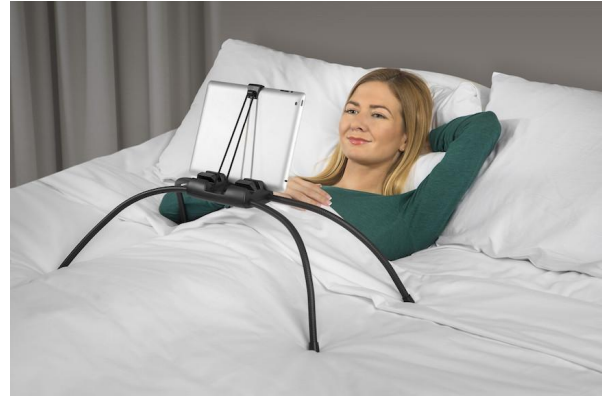

Fig. 6. The Tablift [7]

1. Provides limited space for users to move around

2. Does not provide adequate space to allow optimal comfort in viewing for two people simultaneously

3. Cumbersome to move out of the way in need of getting up

4. Provides some variety of viewing angles, but the number could be increased

The Tablift does not move out of the way as effortlessly as $\mathrm{CN}_{3}$ indicates, and viewing angles are also limited in number, failing $\mathrm{CN}_{4}$.

\subsubsection{Flexible arm}

One concept, which is a close cousin to the TREC-E, is an adjustable arm system made out of thin tubes which can be bent into various shapes, often attached to a desktop with a screw clamp. The adjustable arm allows multiple viewing angles. However, the thin tube does not provide a great stability. When extended to its limits it becomes quite flimsy and difficult to maneuver.

The Stanaway Gooseneck, shown in Fig. 7 is a example of this design an goes for $\$ 15,99$ on amazon.com

Some of its notable features and limitations include:

1. Provides acceptable stability, except at great extension.

2. Does require mounting to a sturdy nearby object.

The Gooseneck most likely has room for improvement when it comes to stability, and also is not overly compatible with furniture, failing $\mathrm{CN}_{1}$.

\section{Design}

Axiomatic Design teaches us that simple and independent features can make for an excellent design. [9]

With a clear focus on functional requirements and their respective design parameters, a products geometry and features can be designed quickly, accurately, and effectively. The coupling between these FRs and DPs is another matter of high importance. This can

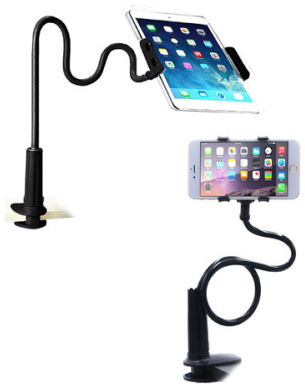

Fig. 7. The Stanaway Gooseneck [8]

be mathematically demonstrated, as is shown in 1 where a non-zero entity represents a connection between the two. If this Cartesian product shows zeros, the corresponding FR and DP are not connected. Should a diagonal line of non-zero values appear, with all other values in the matrix displayed as zeros, then the design can be called un-coupled. This is an ideal design, as changes in one DP do not alter more than the only designated FR. This is called a diagonal matrix, and it satisfies the Independence Axiom: "to maintain the independence of the functional requirements (FRs)" [10].

From the Customer Needs, we built a list of Functional Requirements.

- $\mathrm{FR}_{1}$ Attaches securely between any hard surface and a cushion

- $\mathrm{FR}_{2}$ Holds tablets/smart devices up to 14 inches and $1 \mathrm{~kg}$. One of the largest and heaviest tablets on the market today is the Toshiba Excite 13 [11]

- $\mathrm{FR}_{3}$ Pushes out of the way with minimal force, when the users are in need of getting up

- $\mathrm{FR}_{4}$ Adjusts with minimal force while remaining stable

\subsection{FR-DP Mapping}

The next stage of the design process is to develop lists of design parameters, keeping in mind the Independence Axiom $^{1}$ and the Information Axiom ${ }^{2}$

- $\mathrm{DP}_{1}$ Large mounting plate.

The bracket is designed with a relatively large plate at the bottom end of its first arm which allows it to be attached to any bed or sofa by simply putting the plate under the mattress or sofa cushion.

- $\mathrm{DP}_{2}$ An adjustable clamp grips edge with rubber.

\footnotetext{
${ }^{1}$ Use modularity and avoid coupling

${ }^{2}$ Use robust components or techniques that meet their specifications in a reliable manner.
} 
Table 1. First level FR-DP mapping.

\begin{tabular}{lll}
\hline ID & Functional Requirement & Design Parameter \\
\hline 1 & Fit on furniture & Large baseplate \\
2 & Hold tablet/smart phone & Adjustable grip \\
3 & Push out of way & Bearing on baseplate \\
4 & Adjust height, remain stable & Button to adjust joints \\
\hline
\end{tabular}

The clamp is meant to be equipped with small, adjustable arms so it can grab onto different sizes. The arms need to have high friction surface on the inside to avoid slipping and minimizing clamping force. Analysis in Section 3.2.3 resulted in minimum clamping force of $16,3 \mathrm{~N}$ with friction coefficient of $\mu=0,6$

- $\mathrm{DP}_{3}$ Bearing on a swing arm.

The 1 st arm of the system is going to be vertical and rotate to swing the bracket out of the way.

- $\mathrm{DP}_{4}$ Sturdy yet flexible joints

The joints between arms are going to be very sturdy and rigid to make sure the bracket does not move while in use but they are also going to have the ability to release and become flexible to adjust the viewing angle.

We continue a "zig-zag" procedure to decompose and map the FRs to the DPs as shown in Table 1.

From this mapping we develop the uncoupled design matrix shown in Equation 1

$$
\left\{\begin{array}{l}
\mathrm{FR}_{1} \\
\mathrm{FR}_{2} \\
\mathrm{FR}_{3} \\
\mathrm{FR}_{4}
\end{array}\right\}=\left[\begin{array}{cccc}
X & 0 & 0 & 0 \\
0 & X & 0 & 0 \\
0 & 0 & X & 0 \\
0 & 0 & 0 & X
\end{array}\right]\left\{\begin{array}{l}
\mathrm{DP}_{1} \\
\mathrm{DP}_{2} \\
\mathrm{DP}_{3} \\
\mathrm{DP}_{4}
\end{array}\right\}
$$

\subsection{Analysis}

Online research yielded the results that one of the heaviest tablet available on the consumer market is the Toshiba Excite 13, according to gsmnation.com [11] The Toshiba Excite 13, according to specification from gsmarena. com [12], weighs $998 \mathrm{~g}$. This weight will be used for further analysis.

\subsubsection{Arms}

When choosing material for the arms, it was decided to use aluminum for its low weight and high strength. After searching for vendors, it was found that aluminum tubes were also widely available in many different sizes and for low cost, which confirmed our choice. Other alternatives that were considered were iron tubing, which would have been heavier and carbon fiber tubing which would have been more expensive and more brittle.

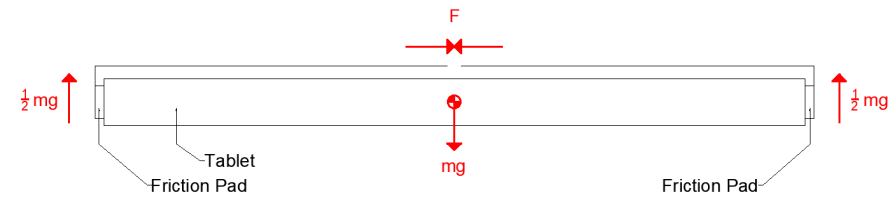

Fig. 8. Forces action on clamp.

The surface finish of the arms would be anodizing the aluminum tubes for a pleasant look and increase toughness. At first, the stand would be available in black, white and silver.

\subsubsection{Joints}

The joints need to survive a bending moment which equals the weight of a tablet at maximum extension. The torque can be calculated as a simple moment arm (Eq 2)

$$
T=m g l
$$

where $T$ is the torque, $m$ is the mass of the tablet and $l$ is the total length of the arm system.

Considering the load from the tablet referenced in Section 3.2 and the arm lengths demonstrated in Section 3.3 results in $T=9.79 \mathrm{~N} \mathrm{~m}$.

\subsubsection{Clamp}

The grip force of the clamp needs to be high enough so that the friction force of the friction pads can hold on to the tablet as illustrated in Fig. 8.

This leads to a simple normal-force friction calculation (Eq. 3):

$$
F=\frac{2 \times \frac{1}{2} m g}{\mu}=\frac{m g}{\mu}
$$

where $F$ is the clamping force, $m$ is the mass of the tablet and $\mu$ is the friction coefficient of the friction pads.

Using a friction factor of 0.6 [13] for rubber and the tablet hanging vertically from the clamp, Eq. 3 results in a $16.3 \mathrm{~N}$ clamping force.

\subsection{CAD}

At the bottom of the unit, there is a large plate that can be slid under a mattress or sofa cushion, see Fig. 9, and connected to that plate is a bearing block with two bearings and two threaded holes which you can use to lock it from rotating. Both parts are made out of plastic because it is light and easy to work with.

As illustrated in Fig. 10 the bearing block is a separate component from the bottom plate and has to be assembled to the bottom plate after a bearing has been inserted. For the threaded holes, there are flanged nuts because plastic threads aren't durable.

The joint, seen in Fig. 11, is 3D printed out of polypropylene. It has a $210^{\circ}$ working angle and 13 possible positions. 


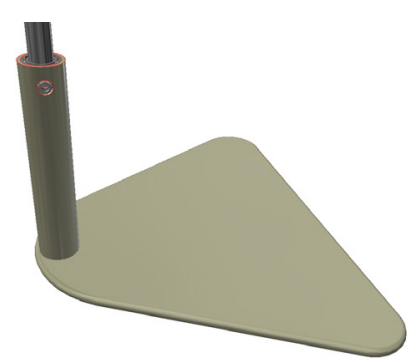

Fig. 9. The bottom plate with the bearing block.

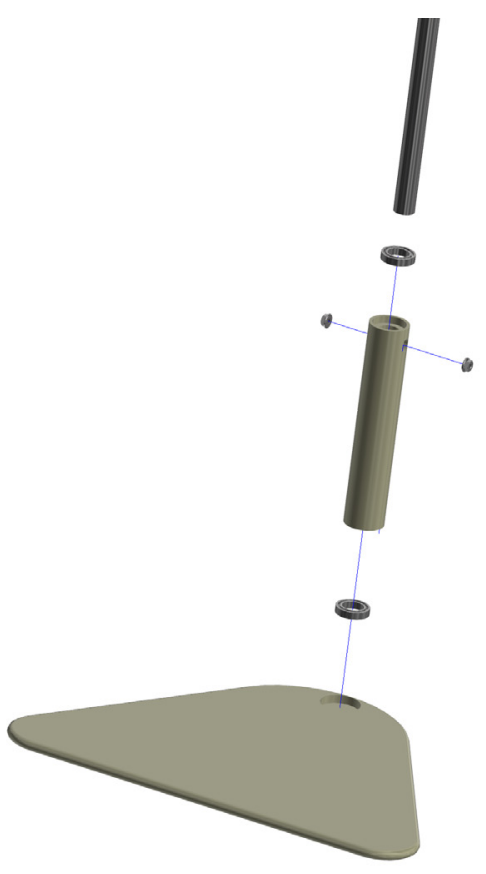

Fig. 10. Assembly presentation of the bearing block.

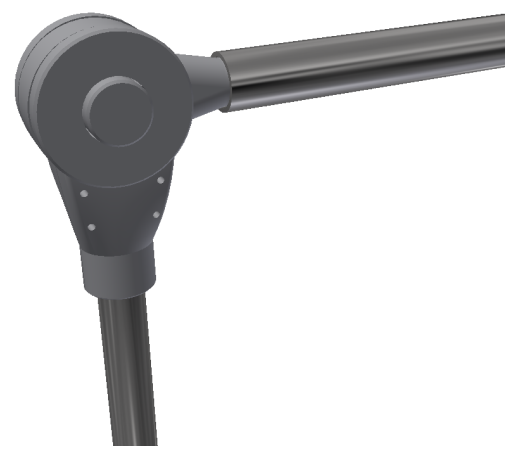

Fig. 11. The joint.

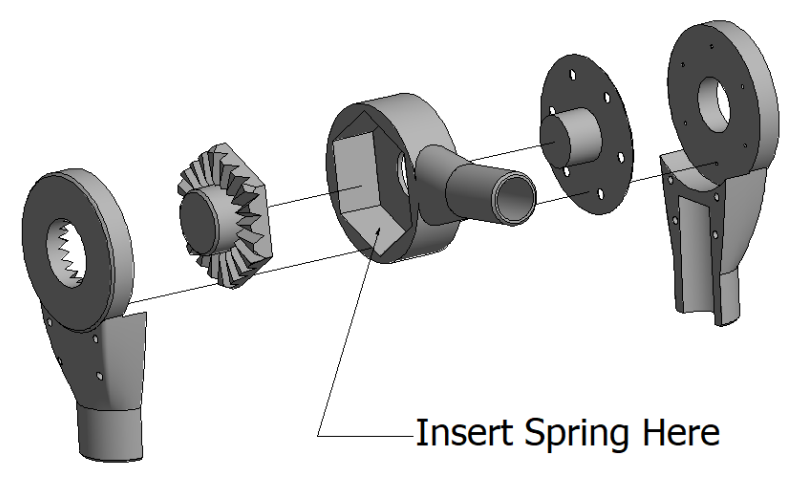

Fig. 12. Explosive view of the joint.

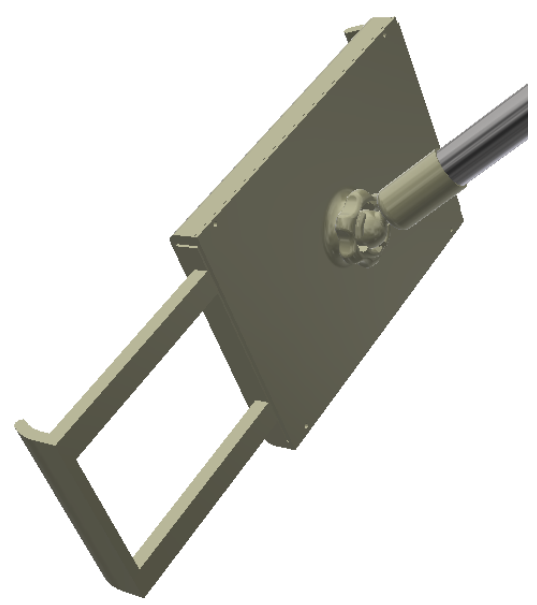

Fig. 13. Clamp and ball joint.

Fig. 12 shows 5 of 6 components of the joint and where a spring (the 6th component) should be installed. The spring makes sure the joint locks in place and by pushing a button on the joints side the spring compresses leaving the joint free to rotate.

Fig. 13 shows the back of the clamp and how it connects to the aluminum tubes with a plastic ball joint.

These components leave us with the final product shown in Fig 14.

The telescopic arm (B+C in Fig. 14) will be around 35 $\mathrm{cm}$ above the face of the user when in horizontal position, with the hight of a mattress of $25 \mathrm{~cm}$ and a head + pillow of $30 \mathrm{~cm}$, the total length of the vertical arm (A in Fig.14) will be $90 \mathrm{~cm}$. The length of the telescopic arm will be adjustable from $50 \mathrm{~cm}$ to $100 \mathrm{~cm}$ for different viewing distances. Finally, the second adjustable arm (D in Fig.14) will be $20 \mathrm{~cm}$ to allow for free adjustments of the ball joint holding the device clamp.

\section{Experiments}

From the start, the team realized that one of the weaker points was the joint between arms A and B. This joint holds a lot of torque, due to the weight of the tablet and 


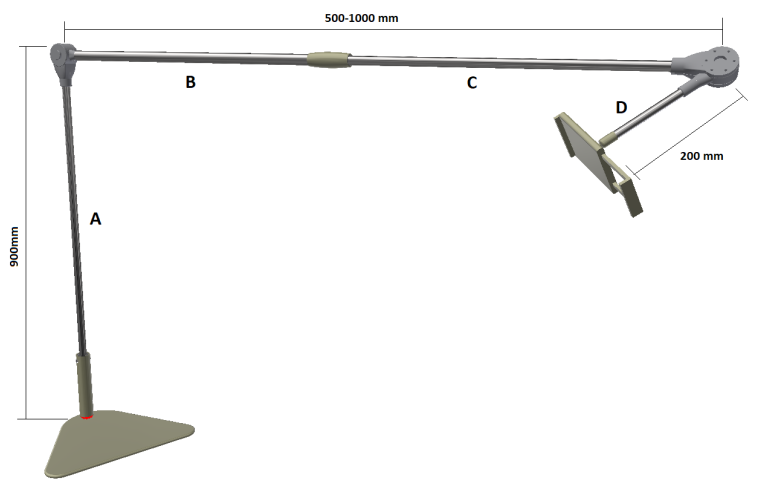

Fig. 14. Assembly of all components with dimensions and labels.

the length of the arm, and a decision was made to test the strength of the joint as soon as a prototype was available.

\subsection{Joint strength test}

The purpose of this test is to evaluate if the 3d-printed adjustment joint prototype is strong enough to hold a tablet safely as analyzed in Section 3.2.2.

For a safety factor of $n=1$, the prototype joint is to be tested by attaching it to a pole and hanging a $1 \mathrm{~kg}$ mass from the pole at a distance of $1 \mathrm{~m}$.

\subsection{Aluminum tube strength/bending test}

The purpose of this test is to evaluate what size of aluminum tubing would be sufficient to: (a) hold the tablet without failing, (b) holding the tablet without bending more than $2 \mathrm{~cm}$ for a $50 \mathrm{~cm}$ long tube and a $1 \mathrm{~kg}$ mass. a

The tubing is to be tested by fixing one end of a $50 \mathrm{~cm}$ tube securely and hanging a $1 \mathrm{~kg}$ mass from the end. The deflection will then be measured. The tube will be fixed to the bottom of a table with a clamp and the bottom of the table used as a reference point. Then the deflection of the tube will be measured before and after a $1 \mathrm{~kg}$ load is applied to the end of the tube. The tubes used will be $20 \times 1 \mathrm{~mm}$ aluminum tubes. These tubes were chosen since they are the tubes in the construction that will be under the most bending moment.

\section{Results and Discussion}

\subsection{Joint strength test}

The joint withstood this experiment, even when the distance was altered to roughly $1.5 \mathrm{~m}$, and excluding the weight of the pole holding the $1 \mathrm{~kg}$ mass.

The conclusion from the test is that the clamp has a safety factor of more than 1 for a $1000 \mathrm{~g}$ tablet at a horizontal distance of $1 \mathrm{~m}$.

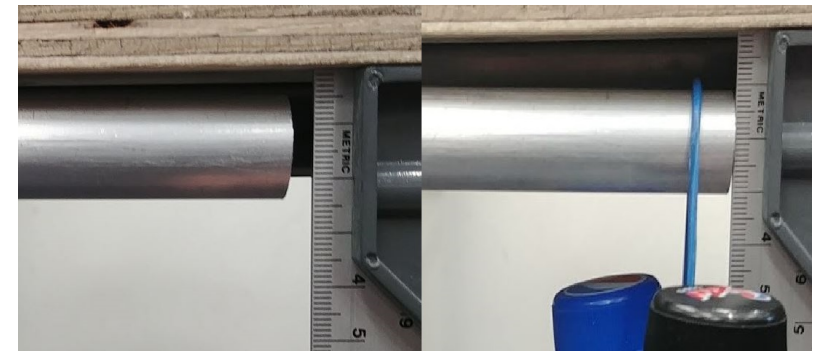

Fig. 15. Deflection of a $50 \mathrm{~cm}$ long, $20 \times 1 \mathrm{~mm}$ aluminum tube. $1 \mathrm{~kg}$ mass at end.

\subsection{Aluminum tube strength/bending test}

A difference in deflection was observed to be $5 \mathrm{~mm}$ as shown in Fig. 15. This deflection is acceptable and will not lead to too much deflection of the stand while in use.

\section{Conclusion}

The TREC-E is a very capable tablet holder. It is powerfully built and can hold up to the heaviest of tablets on the market. It is highly adjustable and can be fitted to most beds and sofas. The FR's that were set at the beginning of the project were met using the DP's.

- $\mathrm{FR}_{1}$ Attaches securely between any hard surface and a cushion

This was solved by using a big baseplate that slides between the cushion of the bed/sofa and the base of the bed/sofa. Even without the mattress on top of the baseplate, stabilizing the holder, it was capable standing upright while holding a device.

- $\mathrm{FR}_{2}$ Holds tablets/smart devices up to 14 inches and $1 \mathrm{~kg}$.

A powerful construction and tests to assure the strength of the design were used to fulfill this FR.

- $\mathrm{FR}_{3}$ Pushes out of the way with minimal force, when the users are in need of getting up

Using a swivel base design with bearings makes sure the TREC-E swivels easily out of the way. A force of $1 \mathrm{~N}$, applied at the middle of the horizontal arm, was enough to swivel the device

- $\mathrm{FR}_{4}$ Adjusts with minimal force while remaining stable

To fulfill this FR, a joint and a ball joint were designed and 3d-printed. This would be made of cast plastic for a mass production which is stronger and has better detail. 


\subsection{Future work}

The next project will be ambitious. The goal is to make a fully autonomous stand that reads what angle the viewers face is and auto adjusts to the perfect viewing angle and distance for a particular device.

\subsection{Summary}

In conclusion, we think that the design was successful. It achieves all of the goals set out at the beginning of the project. The TREC-E is capable of greatly increasing the comfort of viewing media on a smart device in bed or on a sofa.

\section{References}

[1] K.T. Ulrich, S.D. Eppinger, Product Design and Development (McGraw-Hill, 2012)

[2] J. Apter, 'tablet holder" patent, US Patent D732,545, Issued June 23, 2015. Assignee Padcaster LLC.

[3] M. Stevens, B. Augood, “computer tablet holder" patent, US Patent D733147, Issued June 30, 2015. Assignee Kaylor Global, Inc.

[4] D. Girault, “multi media tablet holder” patent, US Patent application 20150009672A1, Applied January 8, 2015.
[5] Sqweezel, patent pending, Sqweezel website, website has been taken down for unspecified reasons as of 2017-08-07., http: //www. sqweezel. com/

[6] Gogo pillow (as seen on tv), https: //www . amazon . co. uk/dp/BOODODDR7C/ref= as_at? slotNum $=4 \&$ ie $=$ UTF $8 \& l$ inkCode $=g 12 \&$ imprToken=WtATkd1-ZYlo74DFEWRr6g\& creativeASIN=BO0DQDDR7C\&tag $=\mathrm{r} 0 \mathrm{dfb} 1-$ $21 \&$ creative $=390957 \&$ camp $=1789$

[7] Tablift, Tablift website, https://www.tablift. com/collections/tablift-universaltablet-stand-all-colors/products/buy-1tablift-for-39-95

[8] Stanaway gooseneck, https://www.amazon.com/ gp/product/B01KC15V1E/ref=s9_acsd_hps_ bw_c_x_1_w

[9] N.P. Suh, Complexity (Oxford University Press, 2005)

[10] N.P. Suh, Axiomatic Design - Advances and Applications (Oxford University Press, 2001)

[11] M. Butler, The Excite 13: The Biggest, Heaviest Tablet in the World, GSMNation Blog (2013)

[12] Toshiba excite 13 at335 (specifications), https://www.gsmarena.com/toshiba_excite_ 13_at335-4691.php

[13] Friction coefficients table, https://www . engineeringtoolbox.com/frictioncoefficients-d_778.html 